\title{
The Structure of Galaxies at Faint Light Levels: Probing Galaxy Assembly
}

\author{
Annette M. N. Ferguson \\ Institute for Astronomy, University of Edinburgh, Blackford Hill, Edinburgh UK EH9 3HJ \\ email:ferguson@roe.ac.uk
}

\begin{abstract}
Many clues about the galaxy assembly process lurk in the faint outer regions of galaxies. Although quantitative study of these parts has been severely limited in the past, breakthroughs are now being made thanks to the combination of wide-area star counts, deep HST imagery and 8-m class spectroscopy. I highlight here some recent progress made on deciphering the fossil record encoded in the outskirts of our nearest large neighbours, M31 and M33.
\end{abstract}

Keywords. galaxies: evolution, galaxies: formation, galaxies: stellar content, galaxies: structure, galaxies: individual (M31, M33)

\section{The Faint Outer Regions of Galaxies: Motivation and History}

The study of the faint outskirts of galaxies has become increasingly important in recent years. From a theoretical perspective, it has been realised that many important clues about the galaxy assembly process should lie buried in these parts. Cosmological simulations of disk galaxy formation incorporating baryons have been carried out by several groups and yield predictions for the large-scale structure and stellar content at large radii-for example, the abundance and nature of stellar substructure and the ubiquity, structure and content of stellar halos and thick disks. These models generally predict a wealth of (sub)structure at levels of $\mu_{V} \sim 30 \mathrm{mag} / \square^{\prime \prime}$ and fainter; their verification thus requires imagery and spectroscopy of galaxies to ultra-faint surface brightness levels.

Since Malin first applied his photographic stacking and amplification technique (e.g. Malin et al. 1983), it has been known that some galaxies possess unusual low surface brightness (LSB) structures-shells, loops, asymmetric envelopes-in their outer regions. Although limited to $\mu_{B} \lesssim 28 \mathrm{mag} / \square^{\prime \prime}$, these images were sufficient to demonstrate that even the most "normal" nearby galaxies could become very abnormal when viewed at low surface brightness (e.g. Weil et al. 1997). Follow-up study of Malin's LSB features, and several more recently-discovered examples (e.g. Shang et al. 1999), has been severely limited due to the technical difficulties associated with detecting and quantifying diffuse light at surface brightnesses $\gtrsim 10$ magnitudes below sky. The currently most viable technique to probe the very low surface brightness regions of galaxies is that of resolved star counts (e.g. Pritchet \& van den Bergh 1994); I review here some recent results from studies of the faint outer regions of M31 and M33.

\section{Substructure Around Galaxies}

Figure 1 shows maps of the red giant branch (RGB) population (age $>1-2$ Gyr) in M31 and M33, covering 40 and 7 square degrees respectively. Although the maps reach the same limiting depth $(\approx 3$ magnitudes below the tip of the RGB), it can be clearly seen that outer regions of M31 contain a wealth of substructure that is not present in M33 (Ferguson et al. 2002, Ferguson et al. 2007, in prep). The faint features visible in the M31 

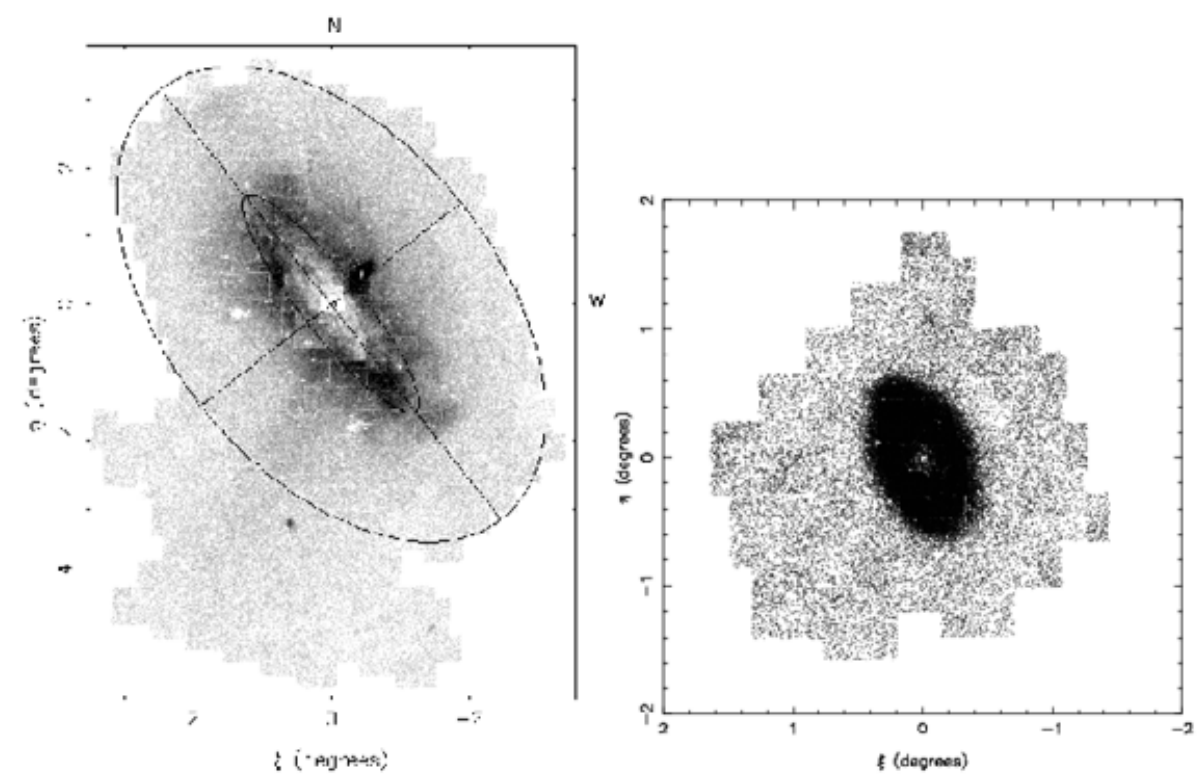

Figure 1. INT/WFC RGB star count maps of M31 (left) and M33 (right). These maps span $125 \times 95 \mathrm{kpc}^{2}$ and $56 \times 56 \mathrm{kpc}^{2}$ on a side respectively.

map have effective V-band surface brightnesses of $\mu_{V} \sim 28-31 \mathrm{mag} / \square^{\prime \prime}$; if substructure is present in M33, it must have surface brightnesses significantly below these values.

Various distinct features can be seen around M31, including a giant stream in the south-east, stellar overdensities at large radii along the major axis, a diffuse structure in the north-east and a loop of stars projected near NGC 205. It is of obvious importance to establish the nature and origin of this substructure. Bullock \& Johnston (2005) predict much substructure in the outer regions of galaxies due to tidal debris from the accretion and disruption of an expected population of $\sim 100-200$ luminous satellites. Gauthier et al. (2006) further show how the accretion of such a satellite population will heat and restructure the thin disk, producing additional "low latitude" debris around galaxies. I summarize here our current understanding of the substructure seen around M31:

- Deep HST/ACS CMDs reaching well below the horizontal branch have been obtained for 8 regions in the far outskirts of M31, including seven regions of visible substructure. Many of the CMDs exhibit different morphologies, suggesting variations in the mean age and metallicity of the constituent stars (Ferguson et al. 2005, see Figure 2). In all cases, the metallicity inferred from the RGB colour is significantly higher $([\mathrm{Fe} / \mathrm{H}] \sim-0.7)$ than that of typical present-day low mass Local Group dwarf satellites $([\mathrm{Fe} / \mathrm{H}] \lesssim-1.5)$, implying that such objects are unlikely to be the progenitors of the observed substructure.

- All substructure fields lying near the major axis contain stars of age $\lesssim 1.5$ Gyr (Faria et al. 2007; Richarsdon et al., in preparation); this includes the NE clump field, which lies at a projected radius of $40 \mathrm{kpc}$. Additionally, the major axis substructure fields are dominated by a strong rotational signature, similar to the HI disk, and a modest velocity dispersion (Ibata et al. 2005). Taken together, these results suggest that the "low-latitude" structure in M31 results primarily from perturbed outer thin disk.

- The combination of line-of-sight distances and radial velocities for stars at various locations along the giant stream constrains the progenitor orbit (e.g. Ibata et al. 2004; Fardal et al. 2006). Currently-favoured orbits do not easily connect the more luminous 
inner satellites (e.g. M32, NGC 205) to the stream however this finding leaves some remarkable coincidences (e.g. the projected alignment on the sky, similar metallicities) yet unexplained. The giant stream is linked to another overdensity, the diffuse feature lying north-east of M31's centre, on the basis of nearly identical CMD morphologies and RGB luminosity functions; orbit calculations suggest this connection is indeed likely.

\section{Diffuse Stellar Halos}

Tidal debris from accretion events will disperse over time and streams from ancient events will have long merged to produce a smooth stellar halo (e.g. Abadi et al. 2005; Bullock \& Johnston et al. 2005). If hierarchical growth is the main mode of mass assembly then such stellar halos should be a generic feature of galaxies. Unfortunately, we have very few observational constraints on the nature and ubiquity of stellar halos around galaxies (see however Zibetti et al. 2004). Even our understanding of the M31 and M33 stellar halos was extremely poor until recently. I summarize some key results here:

\subsection{M31}

Using data from the INT/WFC survey, Irwin et al. (2005) combined diffuse light surface photometry with resolved star counts to probe the minor axis profile of M31 to a radius of $\sim 55 \mathrm{kpc}$ (effective $\left.\mu_{V} \sim 32 \mathrm{mag} / \square^{\prime \prime}\right)$. The profile shows an unexpected flattening (relative to the inner $\mathrm{R}^{1 / 4}$ decline) at a radius of $\sim 30 \mathrm{kpc}$, beyond which it can be described by shallow power-law (index $\approx-2.3$ ), possibly extending out to $150 \mathrm{kpc}$ (Kalirai et al. 2006). This compares favourably with the Milky Way halo, which exhibits a powerlaw index of -3.1 in volume density (Vivas et al. 2006). The discovery of a power-law component which dominates the light at large radii in M31 has profound implications for the interpretation of all prior studies of the M31 "stellar halo". Since these studies generally targetted regions lying within $30 \mathrm{kpc}$ along the minor axis, they most likely probed the extended disk/bulge region of the galaxy and not the true stellar halo.

Keck/DEIMOS spectroscopy has been used to study the kinematics and metallicities of stars in the far outer regions of M31. By windowing out the stars which corotate with the HI disk, Chapman et al. (2006) have detected an underlying metal-poor $([\mathrm{Fe} / \mathrm{H}] \sim-1.4)$, pressure-supported $(\sigma \sim 100 \mathrm{~km} / \mathrm{s})$ component (see also Kalirai et al. 2006). Although it has yet to be proven that this component is the same one which dominates the powerlaw profile at very large radius, the evidence is highly suggestive. Despite many years of thinking otherwise, it thus appears that M31 does indeed have a stellar halo which resembles that of the Milky Way in terms of structure, metallicity and kinematics.

\section{2. $M 33$}

Studies of the M33 stellar halo also had a somewhat checkered history. Mould \& Kristian (1986) measured $[\mathrm{M} / \mathrm{H}] \sim-2.2$ in a field located at $7 \mathrm{kpc}$ along the minor axis of M33 and, for the better part of two decades, this metallicity was generally assumed to reflect that of the M33 halo. Recent work has found a significantly higher metallicity for stars in this same field and, at the same time, suggested that the field is actually dominated by the outer disk and not the stellar halo (e.g. Tiede et al. 2004, Ferguson et al. 2007 in prep). The detection of a power-law structural component in the outskirts of M33 has so far proved elusive, although the RGB clearly becomes narrower and more metal-poor in these parts (Ferguson et al. 2007 in prep). A recent Keck/DEIMOS spectroscopic study has targetted two fields in the outskirts of the galaxy, located at $\sim 9 \mathrm{kpc}$ along the major axis (McConnachie et al. 2006). Although the dominant kinematic component in these fields exhibits strong rotation, there is tentative evidence for an additional low-level 
metal-poor $([\mathrm{Fe} / \mathrm{H}] \sim-1.5)$ component centered at the systemic velocity. While further work is clearly needed to elucidate the nature of this structure, the estimated velocity dispersion $(\sigma \sim 50 \mathrm{~km} / \mathrm{s})$ supports an association with M33's true stellar halo.

\section{Future Outlook}

Quantitative study of the faint outskirts of galaxies provides important insight into the galaxy assembly process. Studies to date have focused on our nearest large neighbours, M31 and M33. While both appear to show evidence for a metal-poor, pressure-supported stellar halo (similar to that of the Milky Way), only M31 shows evidence for recent accretion. In order to put these results in context, we need to establish the properties of a larger sample of galaxies, spanning a range in both host galaxy luminosity and Hubble type. With 8-m class telescopes, it is possible to map the RGB populations in the outskirts of galaxies to distances of $\lesssim 5 \mathrm{Mpc}$; with HST, this work can feasibly be extended to $\gtrsim 10 \mathrm{Mpc}$. Spectroscopic characterization of resolved RGB populations beyond the Local Group is far harder and must await the arrival of 30-m class telescopes.

\section{Acknowledgements}

I thank Scott Chapman, Daniel Faria, Rodrigo Ibata, Mike Irwin, Rachel Johnson, Geraint Lewis, Alan McConnachie and Jenny Richardson for their collaboration. Support from a Marie Curie Excellence Grant (MCEXT-CT-2005-025869) is acknowledged.

\section{References}

Abadi, M. G., Navarro, J. F., \& Steinmetz, M. 2006, MNRAS, 365, 747.

Bullock, J. S., \& Johnston, K. V. 2005, ApJ, 635, 931.

Chapman, S. C., Ibata, R., Lewis, G. F., Ferguson, A. M. N., Irwin, M., McConnachie, A., \& Tanvir, N. 2006, astro-ph/0602604.

Fardal, M. A., Babul, A., Geehan, J. J., \& Guhathakurta, P. 2006, MNRAS, 366, 1012.

Faria, D., Johnson, R., Ferguson, A. M. N., Irwin, M. J., Ibata, R. A., Johnston, K., Lewis, G. F., \& Tanvir, N. R. 2007, submitted.

Ferguson, A. M. N., Irwin, M. J., Ibata, R. A., Lewis, G. F., \& Tanvir, N. R. 2002, AJ, 124, 1452.

Ferguson, A. M. N., Johnson, R. A., Faria, D. C., Irwin, M. J., Ibata, R. A., Johnston, K. V., Lewis, G. F., \& Tanvir, N. R. 2005, ApJL, 622, L109.

Gauthier, J.-R., Dubinski, J., \& Widrow, L. M. 2006, astro-ph/0606015

Ibata, R., Chapman, S., Ferguson, A. M. N., Irwin, M., Lewis, G., \& McConnachie, A. 2004, MNRAS, 351, 117.

Ibata, R., Chapman, S., Ferguson, A. M. N., Lewis, G., Irwin, M., \& Tanvir, N. 2005, ApJ, 634, 287.

Irwin, M. J., Ferguson, A. M. N., Ibata, R. A., Lewis, G. F., \& Tanvir, N. R. 2005, ApJL, 628, L105.

Kalirai, J. S., et al. 2006, ApJ, 648, 389.

Malin, D. F., Quinn, P. J., \& Graham, J. A. 1983, ApJL, 272, L5.

McConnachie, A. W., Chapman, S. C., Ibata, R. A., Ferguson, A. M. N., Irwin, M. J., Lewis, G. F., Tanvir, N. R., \& Martin, N. 2006, ApJL, 647, L25.

Mould, J., \& Kristian, J. 1986, ApJ, 305, 591.

Pritchet, C. J., \& van den Bergh, S. 1994, AJ, 107, 1730.

Shang, Z., et al. 1998, ApJL, 504, L23.

Tiede, G. P., Sarajedini, A., \& Barker, M. K. 2004, AJ, 128, 224.

Vivas, A. K., \& Zinn, R. 2006, AJ, 132, 714.

Weil, M. L., Bland-Hawthorn, J., \& Malin, D. F. 1997, ApJ, 490, 664.

Zibetti, S., White, S. D. M., \& Brinkmann, J. 2004, MNRAS, 347, 556. 


\section{Discussion}

Francoise Combes: We recently found an inner ring in the ISM of M31 with SpitzerIRAC (Block et al. 2006) and the two main rings in the ISM of M31 are nicely interpreted by a head-on collision with M32; so it could be that M32 had several collisions with M31 and contribute also to the other perturbations you presented.

Annette Ferguson: Clearly M32 has been interacting with M31 for some time. However, based on the kinematical data I have discussed, it is difficult at present to connect M32 directlt to any of the outer structures.

Phil Appleton: That was a really nice talk. I just wanted to mention GALEX. We are beginning to see very extended disks in the FUV which go deeper than the deepest optical data (e.g. Cartwheel ring).

Annette Ferguson: The existence of outer star forming regions has been known for some time (e.g. Ferguson et al. 1998). But it's really interesting to hear that underlying older stellar components may be there too.

Claude Carignan: Don't you think that the difference in substructures you see between M31 and M33 is mainly due to the different orientation? M31 being edge-on, there is less crowding problem.

Annette Ferguson: It's certainly true that if we viewed M31 face-on, some of the substructure would project directly on top of the bright disk and be impossible to detect. However, much of the substructure lies beyond the bright disk, and so inclination should not affect its detection. At the surface brightness of the substructure (brighter than 28 mag per square arcsec), crowding is not an issue. 